\title{
Research on the Principle of Optimizing Teaching with Digital Ani- mation in Experimental Teaching
}

\author{
Xiaolin Ma* Mengmeng Zhou \\ Xi'an University of Science and Technology, Xi'an, Shaanxi, 710059, China
}

\section{ARTICLE INFO}

Article history

Received: 21 October 2020

Revised: 30 October 2020

Accepted: 9 April 2021

Published Online: 16 April 2021

Keywords:

Experimental teaching

Digital animation

Intuition

Cognition

Pleasure

\section{Introduction}

As the product of multidimensional static graphics ${ }^{[1]}$, Dynamic graphics pay more attention to information interpretation and transmission. The task of digital animation is to ensure effective communication is better than artistic expression $^{[2]}$. As a form of expression of dynamic graphics, digital animation is also an extension and expansion of dynamic graphic art forms. Besides, digital animation conveys information by simulating reality visualization. Visualization is not a simple beautification of information, but the internal logic and expression of information. Therefore, the awareness of users is key points of digital animation design ${ }^{[3]}$. It is particularly important to establish

\begin{abstract}
With the rapid development of computing technology, experimental teaching explores new teaching methods using virtual laboratory and digital laboratory. Based on the characteristics of visualization, continuity and simulation, digital animation technology has become an important form of application in experimental teaching reform. This paper analyzes the artistic characteristics of digital animation, and summarizes that digital animation can display the experimental information intuitively, enable students to quickly obtain the cognition of the experimental content, and obtain psychological pleasure through digital animation in experimental learning, thus improving the teaching effect of experimental teaching.
\end{abstract}

a good simulation of experimental content and a good experimental thinking mode. Digital animation virtual experiment is to use animation technology to visualize the experimental content through digital media. Its visual characteristics can enable students to clearly and intuitively obtain experimental operation experience and analysis of experimental results under good learning conditions, thus improving the quality of experimental teaching.

\section{The visual display of experimental infor- mation by digital animation}

In general, the human visual reaction time is generally $0.15 \sim 2 \mathrm{~s}$. During the whole process, it takes about $0.4 \mathrm{~s}$

\footnotetext{
*Corresponding Author:

Xiaolin Ma,

Xi'an University of Science and Technology, Xi'an, Shaanxi, 710059, China;

E-mail:1302322628@qq.com.
}

Fund Project:

Research on the Unique Role of Digital Animation in Energy Science Experimental Teaching. 
to focus on the visible target, while it takes $1 \mathrm{~s}$ to identify the target. At the same time, it generally takes $1.5 \sim 2 \mathrm{~s}$ for human eye to analyze the picture and obtain a meaningful perception ${ }^{[4]}$. Experimental operation and experimental phenomenon are often instantaneous processes, which cannot be carefully observed by students in the process of experimental practice. One of the important purposes of digital animation technology is the visual display of information. Digital animation is based on digital technology and animation principle, with model elements and image elements as visual contents. It simulates the real three-dimensional perspective to reproduce the experimental process and experimental results, and describes the experimental phenomena through the changes of object shape and color in the experimental reaction time. For example, in the experiment of adding sodium to water, the combustion of sodium is an instant chemical reaction. Through the actual experimental demonstration, the experimental phenomenon cannot be well observed and the chemical change process of sodium cannot be intuitively understood. However, through digital animation to enlarge and slow down the chemical reaction process of sodium, students can better observe the phenomenon. Digital animation simulates the continuous motion track of the experimental object, such as the dynamic phenomena of those changes in size, position, color, quality, material and other aspects of the experimental object, and uses the change of lens to reproduce the experimental process.

Gestalt psychologists found that under specific conditions, the observation object is organized in a regular and clear way, with the characteristics of symmetry, unity, harmony, simplicity and clarity, which can make the audience obtain more satisfied and pleasant psychological feelings. Therefore, the pursuit of simplicity and perfection is a natural need inherent in vision. When human vision observes things, it will have a strong trend to change the observed objects. On the one hand, it will enlarge and expand those related features; on the other hand, it will cancel and ignore those good Gestalt features that prevent them from being concise and regular ${ }^{[5]}$. Digital animation can show the instruments, equipment and materials needed in the experiment in the most concise and clear form. In the design, the adverse factors including light irradiation, equipment blocking, background environment visual influence and the like in the actual experiment are eliminated, so that students can obtain more effective and direct observation effects in the experimental demonstration process. For example, in the practical operation of the experiment, due to those influence of the light irradiation angle, the visual influence of the equipment and the different positions of the operator, the visual effects will be different, which will directly affect the accuracy of the experimental results or experimental data. Through the display and simulation of digital animation technology, these unfavorable factors are eliminated, and the operator can simply and clearly organize the most useful and effective experimental observation and operation to obtain valuable information to the greatest extent. This can meet the inner needs of the audience, realize the greatest degree of knowledge satisfaction, and improve the teaching results of experimental teaching.

In the process of experimental practice, the experimental process is complicated. Besides, the experimental phenomena change rapidly and are affected by many factors. For example, the negligence of the operator and the change of the experimental environment will affect the recording of experimental data, the observation of experimental phenomena and the effective comparison of results. Due to its timeliness of content and directness of vision, as well as the editable function of process animation, digital animation technology can simulate and complete the experiment in the experimental information, results and operation. In addition, at the same time, it can analyze the whole experimental process by recording experimental data, summarizing image data, and effectively display it with visual graphics in real time. Experimental data often show scattered characteristics. Digital animation is utilized to show complex experimental information concisely, accurately and truly, so the experimental information, results and data are clearly displayed at a glance. It improves the learning efficiency of students and enhances the timeliness of experimental teaching.

\section{Students' Quick Cognition of Experimental Contents}

Digital animation relies on models and images to complete the transmission of information. It aims to effectively display the complicated experimental process with real, vivid and concise pictures, to quickly convey the experimental information content through intuitive images and lenses, thus enhancing students' cognition. With such artistry in itself, it can also enhance students' artistic appeal. The living habits, educational level, knowledge and experience of students vary, so their abilities to accept things are also different. However, as an art of image and lens, digital animation art is an art form without borders. Compared with static graphics and text transmission, digital animation can enable students to recognize the experimental cases more effectively, receive and comprehend the experimental contents quickly and effectively.

In the process of information transmission, the audi- 
ence usually first notice the motion phenomenon of the image, and keep continuous attention to it, selecting effective information for acceptance and memory. Dynamic pictures can rely on their motion speed to guide the audience's motion vision to examine the track, so that they can track the images according to the designer's established route and spend more time on the key information of the images $^{[6]}$. The biggest advantage of digital animation lies in the dynamic nature of the pictures and the continuity of the lens. The students' learning process through watching is continuous, at the same time, they can also be attracted by continuous dynamic elements, thus enhancing their attention. Gestalt theory holds that when people perceive things, they treat objects as a whole rather than the sum of individual phenomena. The whole perceived by users is not only composed of each single element, but also related to the internal characteristics between elements. Moreover, Users tend to perceive the content things as simple, orderly and complete as possible ${ }^{[7]}$. Scientific experiment is a continuous practical activity that features continuity and instantaneity. In experimental teaching activities, the information images obtained by students in written still words and pictures will soon disappear in memory without effective continuity and extension, thus increasing the cognitive difficulty of experimental knowledge. Digital animation can continuously express the content in a certain period of time, enabling students to obtain information quickly, effectively in enough times, thus strengthening indoor teaching content and improving learning effect.

People's vision is the superposition of time and speed, and the continuous continuity and superposition of information and digital animation can rely on the timeline to integrate images, sounds and characters to convey information to the audience. In the process of information transmission, the audience will first notice the phenomena of graphic movements and keep continuous attention to it under the influence of people's persistence of version, thus selecting effective information for acceptance and memory ${ }^{[8]}$. This a process of information superposition. Based on the picture information of the current and previous frame, a complete information chain is formed. As a visual expression form with time dimension, digital animation can be changed in accordance with a certain preset process in the transmission process. The audience only needs to organize the graphics, characters, sounds and other elements contained in these dynamic graphics according to logical thinking ${ }^{[9]}$. For example, when studying the knowledge explanation of parabola of objects, if there is only a static picture, it is difficult for students to understand the falling phenomenon of parabolic arc caused by the earth's gravity, and they cannot really master the prin- ciple of gravity of the falling phenomenon. Following the lens of digital animation, students can form a whole of experimental phenomena with continuous pictures, display the consistency of information, and improve the degree of information screening and refining, thus concentrating on the key information of graphics for more time. Therefore, digital animation plays the role in information strengthening.

\section{Psychological Pleasure Generated by Ani- mation in the Process of Experimental Teach- ing}

The form of digital animation belongs to the category of art that is processed artistically. Like other artistic forms, it has strong visual aesthetic feeling. With both artistic expression and beautiful communication form, the process of information expression can more strongly stimulate students' instinctive cognitive desire, behavior and satisfaction in the process of teaching and learning, thus achieving effective communication and information transmission for experimental teaching. For example, with colorful graphics showing its content and aesthetic visual form showing its appearance, the current food packaging can effectively stimulate buyers, thus improving buyers' purchasing desire. Another example is that when people meet relatives and friends, the emotional form of hugging will be better than the verbal expression. Experimental teaching activities are rigorous, so it is natural for students to have boring and passive learning mentality in the process of learning and operation. Digital animation conveys experimental information with aesthetic vision. When students receive knowledge information, they are in a relaxed and pleasant learning state, which makes experimental learning activities complete in an active learning state.

Digital animation is not only the demonstration of experimental teaching and the simulation of experimental process, but also the comparison of experimental results and the reflection of experimental effects. With the characteristics of time, the experimental process is a continuous reaction of instantaneous phenomena. Experimental teaching activities are deep thinking interwoven with knowledge, consciousness, emotion and thought. In traditional experimental teaching methods, the phenomena generated by experimental operation are instantaneous, students do not have enough time to observe and think about them. At the same time, the experimental results are the final phenomenon of each paragraph of the experiment, which does not show the process well and students cannot think effectively in a short period of time. Students often fell a certain sense of pressure and tension. For ex- 
ample, there is a chapter in Journey to the West, which tells a story named "Zhu Bajie eats Ginseng fruit". In that story, as greedy man, Zhu Bajie directly swallowed a stolen ginseng fruit without any taste of it. The same is true of experimental learning. Since experimental learning is instantaneous and irreversible, it fails to leave enough time for students to analyze and think. However, digital animation can effectively reduce negative effects, meet students' both learning and psychological needs, and improve the teaching effect of experimental teaching through its artistic treatments.

\section{Conclusion}

Experimental teaching is an important teaching link in engineering disciplines. The teaching effects and acceptance of experimental teaching contents will directly affect the understanding and application ability of subject knowledge. Colleges and universities across the country attach increasing importance to the reform and innovation of experimental teaching. In the reform of experimental teaching methods, digital animation has visual features of intuition and aesthetic nature, and technical features of interaction and simulation, which have been highly recognized by relevant scholars, teachers and students. The optimization effect of digital animation on experimental teaching will also be paid attention by an increasing number of researchers.

\section{References}

[1] Zhou Pengcheng. Research on Source Design Expression of Dynamic Graphics in Outdoor Digital Media [J]. Decoration, 2016(8):142-143.

[2] Ian Crook, Peter Beer. Fundamentals of Dynamic Graphic Design: From Theory to Practice [M]. Beijing: China Youth Publishing House, 2016:154-155.

[3] Wu Zhendong, Guo Weimin, Ai Xiaoqun. Research on Problem-oriented Teaching Mode of Dynamic Graphic Design [J]. Decoration, 2016(10):109-112.

[4] Xin Yong, Li Xin. Application of Visual Principle in Highway Design [J]. Zhongnan Highway Engineering, 2006,08(31): 86-88.

[5] Zhao Ziyong. Application of Visual Principle in Teaching [J]. Teaching and Management,1998(78): 61-21.

[6] Ye Huiling. Graphic Expression of Graphic Design [D].Hangzhou: China Academy of Fine Arts, 2010.

[7] Miao Dongxin, Yu Shulan. Application of Gestalt Theory in Data Visualization Design[J]. Art Science and Technology,2020:80-96.

[8] Gong Yi. Research on the Transmission of Dynamic Graphic Information in Art Design [J]. Packaging Engineering,2016,9(18):211-214.

[9] Wade Mack. Graphic Design Beyond Ordinary [Z]. Posts \& Telecom Press Co., LTD, 2010. 